\title{
Preface
}

\section{Mini-Forum: Roles of Amyloid- $\beta$ and Tau Phosphorylation in Neuronal Repair and Protection}

\author{
Garth Bissette \\ Department of Psychiatry and Human Behavior, University of Mississippi Medical Center, Jackson, MS, USA
}

This mini-forum is devoted to the hypothesis that the earliest alterations in amyloid- $\beta(\mathrm{A} \beta)$ and hyperphosphorylated tau protein are produced by cellular machinery to protect against damage or in an attempt to repair damage that has occurred. The contributors to this issue have expertise in various disciplines that address the components of Alzheimer's disease (AD) from both clinical and basic science research perspectives. In the first article, I review the data for increased production of either $\mathrm{A} \beta$ or phosphorylated tau protein in response to such diverse stimuli as stimulant drugs, head injury, certain inhaled anesthetic drugs and responses of the hypothalamic-pituitary-adrenal axis in AD.

Koudinov and colleagues review data that supports their contention that most of the observed alterations in clinical AD are attempts to return cellular homeostasis to normal after disruption by physiological mechanisms that restore plasticity. They view the increased pathologic protein production and biochemical alterations as secondary responses to an as yet unknown etiology for development of $\mathrm{AD}$ and argue that these components are not useful therapeutic targets.

Hiltunen, van Groen, and Jolkonnen present data from animal models with mutant or normal amyloid$\beta$ protein precursor $(\mathrm{A} \beta \mathrm{PP})$ and their responses to ischemic insult. Transgenic mice with mutant human $\mathrm{A} \beta \mathrm{PP}$ have poor outcomes after ischemia relative to those with normal human $\mathrm{A} \beta \mathrm{PP}$ and the overexpression of normal hA $\beta \mathrm{PP}$ provides a degree of protection against the sequelae of ischemic insult.
Blennow and Zetterberg review the evidence for using $\mathrm{A} \beta$ and phosphorylated tau in cerebrospinal fluid as a biomarker for AD relative to other dementias and their potential use for early diagnosis. Transient alterations in these proteins after head injury indicate that the neurodegeneration and dementia associated with $\mathrm{AD}$ is not always a result of increased $\mathrm{A} \beta$ and phosphorylated tau production.

Menon, Sattel, and Jolles examine the limitations of neuroimaging in differentiating vascular dementia from $\mathrm{AD}$ and the similarity in the various imaging techniques applied clinically to diagnose AD and vascular dementia. Functional and structural imaging techniques may not differentiate these very distinct etiologies as well as PET or spectroscopy.

Kadish and van Groen review data indicating that lesions of the entorhinal cortex in normal, aged, and transgenic mice produces different responses, with aged mice showing less regeneration after lesion and the presence of $\mathrm{A} \beta$ deposits in transgenic mice does not prevent post-lesion sprouting. They observe increased regeneration in presenilin mutant mice but no increased regeneration in mice overexpressing $\mathrm{A} \beta$ leading them to conclude that the presence of $\mathrm{A} \beta$ does not prevent attempts at neuronal repair.

Castellani and colleagues review the evidence supporting the view that proteinopathies, including $\mathrm{A} \beta$ and hyperphosphorylated tau protein, in AD reflect downstream components of a process that begins with earlier alterations in responses to oxidative stress, inflam- 
mation, and alterations in the cell cycle. They also argue that these proteinopathies are not useful therapeutic targets for preventing neurodegeneration and dementia.

The last two contributions examine the responses of $\mathrm{A} \beta$ and tau protein hyperphosphorylation, respectively, to mice exposes to different stressors.

Rissman reviews the data that tau phosphorylation is important in dendritic remodeling after transient stress such as restraint. The effects of restraint on tau phosphorylation are dependent upon the type-1 corticotropin-releasing factor receptor as demonstrated by selective knockouts and receptor agonist and antagonists.

Hong and Csernansky examine the effects of isolation stress on $\mathrm{A} \beta$ production in mice with the Swedish mutation in $\mathrm{A} \beta \mathrm{PP}$. They also find that this effect depends upon the type-1 CRF receptor and that an in- tact hypothalamic-pituitary-adrenal axis is required for successful induction of $\mathrm{A} \beta$ after isolation stress.

These papers together adumbrate a role for both $\mathrm{A} \beta$ and hyperphosphorylated tau in mediating cellular responses to disturbances in homeostasis. Conditions that require neural sprouting, neurite growth or return to biochemical equilibrium often induce the production of one or both of these "pathologic" proteins that accumulate in the brain of AD subjects. That these developments occur in conditions that are not part of the classic definition of AD indicate their importance in normal brain functions and caution against reflexive attempts at neutralization of these putatively beneficial proteins. These papers also re-emphasize the importance of further research into the actual process(es) that allow these initial repair and protection efforts to fail in the evolution of actual AD. 